\title{
Performance and immune characteristics of bronchoalveolar lavage by research bronchoscopy in pulmonary tuberculosis and other lung diseases in the Western Cape, South Africa
}

Carly Young ${ }^{\dagger}$, Petri Ahlers ${ }^{\dagger}$, Andriette M. Hiemstra, Andre G. Loxton, Andrea Gutschmidt, Stephanus T. Malherbe, Gerhard Walzl, Nelita Du Plessis ${ }^{*}$ (D) and the SU-IRG consortium

\begin{abstract}
Background: Tuberculosis (TB) remains a debilitating, deadly disease that warrants innovative research tools to fully understand the pathogenesis and host immune responses, particularly at the site of infection and disease. In this regard, bronchoscopies with bronchoalveolar lavage (BAL) serve as a valuable technique for site of disease sample retrieval for further clinical- and basic research. Here we investigate the feasibility of research bronchoscopies in a low/middle-income area, where TB remains rife, and assess the value of retrieved BAL cells (BALC) for downstream fluorescent-based cellular evaluations.

Methods: Using quantitative and qualitative methods, we evaluate the outcomes, safety, tolerability, participant -perception and -experience, while also providing insight into participant recruitment and screening processes of our study. Using light microscopy differential counting for BALC analysis, we evaluate the cellular composition of BAL fluid (BALF) from TB patients, healthy community controls and patients with other lung diseases. We also use flow cytometry to describe the challenges associated with fluorescence-based phenotypic analysis of autofluorescent BALC.

Results: Our findings suggest that research bronchoscopies are safe, acceptable procedures for research participants and are indeed a feasible technique for future study design. We also suggest that the majority of participants are receptive to the proposition of a second research bronchoscopy. This poses an important avenue for research entailing follow-up investigations of the same study participant. Furthermore, our results show that smoking is characterized by retrieval of BALC containing particulate matter, that interferes with fluorescence-based flow cytometry data analysis. Based on light microscopy differential cell counting, our findings suggest that there are differences in the cell yields and cellular composition of the BALF between TB patients, healthy community controls and patients with other lung diseases. We also report on subject characteristics and demographic factors, namely gender and age, that have the potential to affect cell yields and cellular data of BALF.

(Continued on next page)
\end{abstract}

\footnotetext{
* Correspondence: nelita@sun.ac.za

${ }^{\dagger}$ Carly Young and Petri Ahlers contributed equally to this work.

DST-NRF Centre of Excellence for Biomedical Tuberculosis Research, South African Medical Research Council Centre for Tuberculosis Research, Division of Molecular Biology and Human Genetics, Faculty of Medicine and Health Sciences, Stellenbosch University, Cape Town, South Africa
}

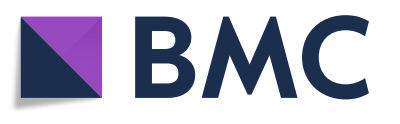

(c) The Author(s). 2019 Open Access This article is distributed under the terms of the Creative Commons Attribution 4.0 International License (http://creativecommons.org/licenses/by/4.0/), which permits unrestricted use, distribution, and reproduction in any medium, provided you give appropriate credit to the original author(s) and the source, provide a link to the Creative Commons license, and indicate if changes were made. The Creative Commons Public Domain Dedication waiver (http://creativecommons.org/publicdomain/zero/1.0/) applies to the data made available in this article, unless otherwise stated. 
(Continued from previous page)

Conclusions: These findings will serve as a valuable reference for appropriate planning and design of studies involving clinical bronchoscopies for TB and lung disease research.

Keywords: Bronchoalveolar lavage, Tuberculosis, Lung disease, Bronchoscopy

\section{Introduction}

Bronchoscopies are widely performed as part of the clinical workup of lung disease, including the diagnosis and grading of cancerous lung lesions and identification of lung pathogens. It is thought that the cellular patterns in bronchoalveolar lavage (BAL) fluid (BALF) also reflect immune processes and host responses within the lung airways, hence its increasing popularity in the pulmonary research field [1-5]. In this way, BAL samples allow researchers to investigate cellular responses directly from the affected region with relatively low to moderate risk, to gain a better understanding of the underlying pathophysiological mechanisms of lung disease.

Tuberculosis (TB) remains the leading cause of death due to infectious disease, caused by the Mycobacterium tuberculosis (Mtb) bacilli [6]. Mtb is most widely known for manifesting in the lung, leading to pulmonary $\mathrm{TB}$ (PTB) disease. For this reason, BAL procedures are of particular interest to TB researchers, however, relatively little has been published in this regard, compared to more easily accessible sample types such as blood, saliva, sputum and urine. Although bronchoscopy has common diagnostic or therapeutic indications, it is perceived as less acceptable when performed for research purposes, with no direct patient benefit.

Despite being considered as a safe procedure when performed by a skilled professional in the absence of contra-indications, bronchoscopies remain an invasive technique necessitating operator proficiency, established operating procedures that include safety measures and well-equipped facilities. A study by Poi and colleagues reported that $58 \%$ of study participants expressed fear of undergoing bronchoscopy [7]. However, another research bronchoscopy study demonstrated that the procedure was well-tolerated and did not negatively influence participants' future decisions regarding health care-seeking behavior [8]. A review by Martinsen et al., evaluating research bronchoscopy participation, identified seven relevant studies, of which six were conducted in Europe. This highlighted the need for further insight into subject participation in research bronchoscopy studies, particularly in low to middle-income countries such as South Africa [9].

In the current study, we address the clinical feasibility and practical aspects concerning research bronchoscopies in the Western Cape, a highly TB burdened region in South Africa. Considering the importance for research bronchoscopies to be time- and cost-effective, safe and acceptable to the population of interest, we evaluate the safety, tolerability, participant -perception and -experience, while also providing insight into participant recruitment and screening processes. We summarize our assessment of the feasibility of research bronchoscopies and provide expected reference ranges for BAL cell (BALC) and BALF retrieval and composition from TB patients, healthy community controls and patients suffering from non-TB related lung disease in the context of the social, economic, and health setting of the Western Cape region, a setting with both affluent and poor socio-economic societies. Lastly, we discuss problems associated with fluorescence-based phenotyping of BALC from smoking participants.

\section{Materials and methods \\ Study population}

Study participants were HIV-infected and uninfected adults between the ages of 18 and 80, who volunteered to be enrolled in a research bronchoscopy study from 2016 to 2018. All healthy community controls ("healthy" group), participants in close contact with an active TB patient ("TB contacts") and all but one of the PTB patients ("TB cases") were HIV-uninfected individuals. The HIVinfected TB case presented with extra-pulmonary TB.

All active TB cases, TB contacts and healthy community controls underwent a chest $\mathrm{x}$-ray (CXR) as part of the initial screening. Only participants with CXR findings that did not preclude unsafe performance of bronchoscopy, a Karnofsky score of $>60 \%$, no evidence of severe aneamia $(\mathrm{Hb}>9 \mathrm{~g} / \mathrm{dl})$ and no serious systemic condition that, in the opinion of the study clinician, would make the performance of a bronchoscopy unsafe were included in the study. Active TB cases presented with clinical, culture, GeneXpert and radiographic results characteristic of pulmonary $\mathrm{TB}$ disease. At the time of bronchoscopy, TB cases received less than seven doses of anti-tuberculosis treatment. Healthy community control subjects were recruited from the same communities as TB cases but had no clinical, radiological or mycobacterial evidence of TB disease or other diseases detected by clinical examination, medical history or by screening medical tests, that included a fingerprick random glucose and hemoglobin test. TB contacts included participants with close contact with an active PTB patient for at least 1 month prior to TB diagnosis and 
included participants with or without diabetes mellitus (DM). The subset of participants with DM (confirmed by $\mathrm{HbA} 1 \mathrm{c} \geq 7 \%$ ) included in the TB contacts group, had no history of a recent myocardial infarction or any signs of myocardial ischemia on electrocardiogram (ECG).

TB cases, TB contacts and healthy community controls had no clinical indication for bronchoscopy and bronchoscopies were done for research purposes only. Other lung disease (OLD) participants were recruited from the Pulmonology Division of Tygerberg Academic Hospital (TBH) and had clinical indications for bronchoscopy. OLD participants presented with a variety of respiratory conditions including, lung infiltrations, unidentified lesions, sarcoidosis or other symptoms of respiratory disease.

Clinical cases were not included in the bronchoscopy procedure discussion, since they had clinical indications for bronchoscopy and the procedure was performed and monitored by TBH staff, rather than our research clinicians. Analysis and discussion of BALC data included the OLD cohort and a subset of TB participants and healthy community controls (Fig. 1). Written informed consent was obtained from participants and study design was approved by the Stellenbosch University Ethics Review Committee (IRB number N16/05/070 and N13/ 05/064).

\section{Bronchoscopy procedure}

The bronchoscopy procedure was standardized for all research participants, derived from the British Thoracic society guidelines on diagnostic flexible bronchoscopy [10]. Bronchoscopies were performed by a skilled endoscopists in the bronchoscopy theatre of a tertiary hospital in the Western Cape Province of South Africa, Tygerberg Hospital. All participants received topical lignocaine spray in the larynx and nasal mucosa, and were

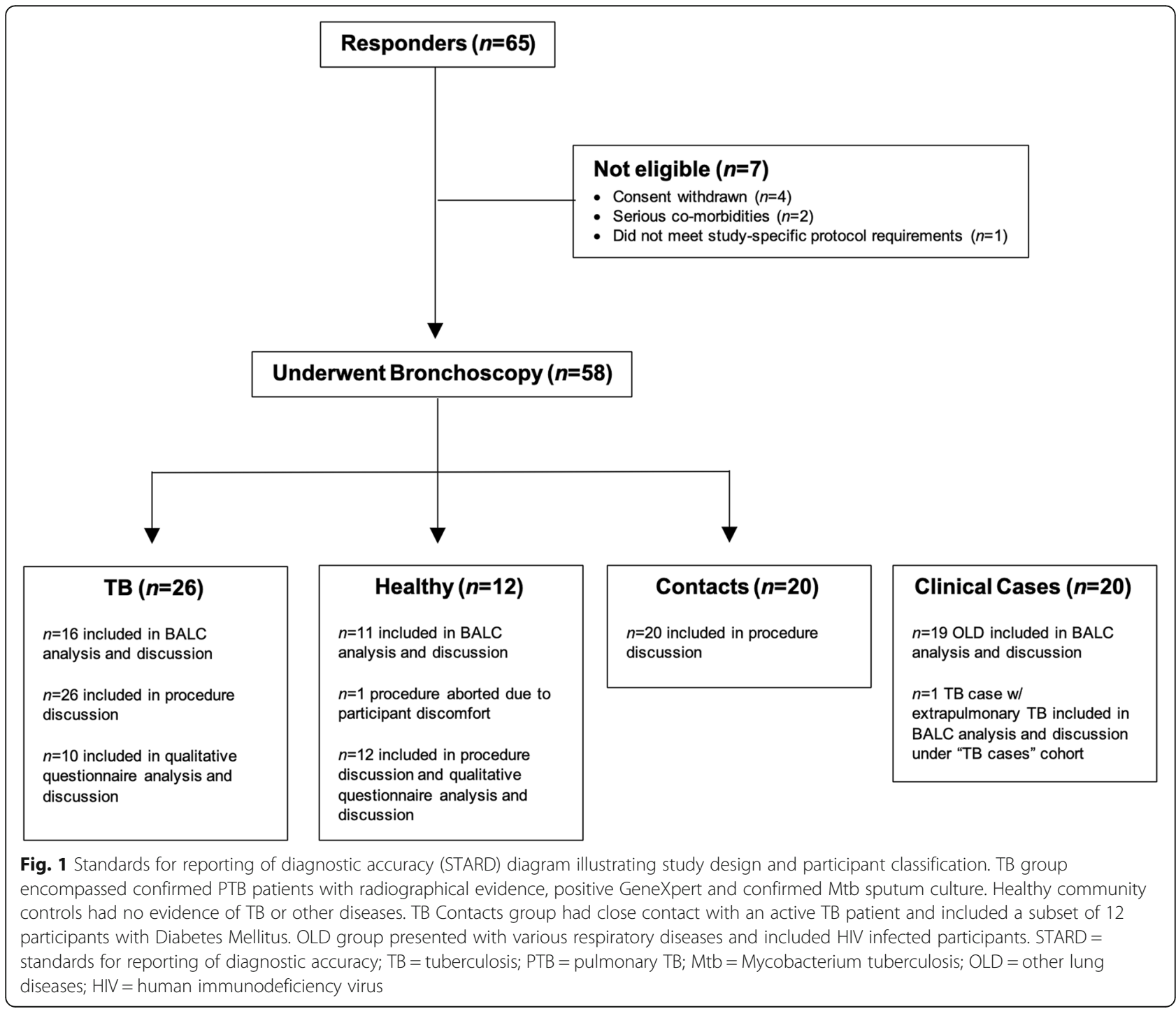


administered intravenous sedatives for conscious sedation. Sedative drugs included midazolam, propofol and fentanyl, administered on their own or in combination. Study participants received remuneration of R400 ( 30US\$) to compensate for loss of time and income. Participants received a telephonic follow up call at $72 \mathrm{~h}$ and 14 days post bronchoscopy by our research clinicians, but did not apply to the OLD cohort since these participants had clinical indications for bronchoscopies and were followed up by $\mathrm{TBH}$ resident clinicians for their underlying condition.

\section{Bronchoalveolar lavage}

BALF samples were obtained according to international guidelines [11]. The bronchoscope was inserted through either nostril or the oral route. Topical lignocaine was sprayed onto the vocal cords and tracheal bifurcation. The bronchoscope was targeted to an identified region of the lung presenting with abnormal or pathologically suspicious lesions, according to radiological imaging. Healthy community controls and TB contacts had no detectable pulmonary lesions according to imaging and the bronchoscope was targeted to lung regions affording ease of accessibility. The lung segment was lavaged by instilling sterile saline solution at $37{ }^{\circ} \mathrm{C}$ up to a maximum volume of $300 \mathrm{ml}$ in aliquots of $60 \mathrm{ml}$ at a time, with immediate aspiration between aliquots. TB contacts received a maximum volume of $200 \mathrm{ml}$ instilled saline due to specific protocol specifications. Aspirated fluid retrieved from the BAL procedure was collected in sterile $50 \mathrm{ml}$ polypropylene tubes and transported on ice immediately to the laboratory, were BALF samples were processed within $2 \mathrm{~h}$ of collection.

\section{BALF processing and cell isolation}

BALC were isolated using centrifugation for $7 \mathrm{~min}$ at $300 \times \mathrm{g}$ following sterile filtration (Falcon ${ }^{\circ} 70 \mu \mathrm{m}$ Cell Strainer; Corning Inc., NY). Cells were washed twice using Phosphate Buffered Saline (PBS) for $5 \mathrm{~min}$ at $300 \times \mathrm{g}$ and viability and cell count determined by Trypan Blue exclusion method [12].

\section{Differential BALC counts}

A differential cell count of total BALC was performed as previously described with minor modifications [13]. Briefly, isolated suspensions containing 50,000 BALC was placed on a multi-well microscope slide (Miltitest Slide, 8 well; MP Biomedicals, LLC, Santa Ana, CA) covered with Whatman 50 filter paper. Cells were left to adhere to the slide surface overnight and stained using Rapidiff Stain Set according to manufacturer's recommendations. A total of 300-400 cells were manually counted by light microscopy (Zeiss Axioskop 2). Cells were classified according to their complexity of morphological appearances.

Analysis of innate autofluorescent cells by flow cytometry Approximately $3 \times 10^{6}$ total BALC from a subset of participants were stained with antibodies for cell surface receptors to discriminate innate immune cell lineages by flow cytometry. Briefly, selected antibodies were titrated to identify the best performing concentration. Compensation and staining controls (fluorescence-minus-one) were included to account for fluorescence spill-over and correctly determine positively and negatively stained cell populations. Controls were prepared using both BALC and BDCompBead Plus (Becton Dickinson, New Jersey, USA) for larger cell types. BALC samples were incubated with fluorescence-conjugated monoclonal antibodies against CD11b-PerCP and CD33-PE (Becton Dickinson) for $20 \mathrm{~min}$ in $50 \mu \mathrm{l} \mathrm{FACS}$ buffer containing PBS with $5 \%$ fetal calf serum at room temperature and subsequently washed twice with FACS buffer. These incubations were performed with or without a quenching procedure using cellular incubation ( $5 \mathrm{~min}$ on ice) with a crystal violet solution $(2 \mathrm{mg} / \mathrm{ml}$ in sodium chloride), before staining and cell membrane permeabilization (eBiosciences 10x Permeabilization Buffer; Thermo Fisher Scientific Inc., Waltham, MA), as described previously [14]. Stained BALC were fixed in $4 \%$ formaldehyde and immunofluorescence signal acquired on a BD FACSCantoII cytometer using the BD FACSDiva software, version 6.1.2 (Becton Dickinson). A minimum of 100,000 events were recorded during sample acquisition and FCS data analyzed using FlowJo software (version 10.1, Oregon, USA).

\section{Statistical analysis}

Statistical analysis was performed using Statistica 12.0 and GraphPad Prism 7.0 software, with Fisher's exact test to examine non-random associations between categorical variables where appropriate. One-way analysis of variance (ANOVA) and analysis of co-variance (ANCOVA) tests (parametric or non-parametric as appropriate after normality test and Levene's test for homogeneity of variance) with Fisher's least significant difference (LSD) as post host for all pairwise comparisons, were used to compare variables between groups where suitable. Pearson's correlation or Spearman's rank-order correlation tests (as appropriate following normality test) were used to determine correlative relationships between investigated variables.

\section{Results}

\section{Patient characteristics}

A total of 65 participants initially agreed to undergo research bronchoscopies, of which 58 underwent the procedure (Fig. 1). Of the seven participants that did not 
undergo the bronchoscopy, four withdrew consent (three on the day of planned procedure and one prior) and three were excluded by the clinical team (two due to serious co-morbidities and one did not meet study-specific protocol requirements). Of the 58 participants that underwent bronchoscopy, 26 were TB cases, 12 healthy community controls and $20 \mathrm{~TB}$ contacts, 12 of which had DM (Fig. 1). Analysis of isolated BALC pertain to information collected from samples of a subset of 11 healthy community controls, all OLD participants and $17 \mathrm{~TB}$ cases, whose characteristics are described in Table 1.

Table 2 describes intravenous sedation doses, bronchoscopy duration and incidental findings at the time of bronchoscopy for TB, healthy community control, TB contacts and a combination of all cohorts. Sedatives used during bronchoscopies were midazolam, fentanyl and propofol, used in isolation or in combination, as described in Table 2. The choice of sedative(s) used depended on the clinician's preference, study specific protocol requirements and participants characteristics. All except two cases received either midazolam in

Table 1 Subject characteristics

\begin{tabular}{|c|c|c|c|}
\hline & $\begin{array}{l}\text { TB } \\
(n=17)\end{array}$ & $\begin{array}{l}\text { Healthy } \\
(n=11)\end{array}$ & $\begin{array}{l}\text { OLD } \\
(n=19)\end{array}$ \\
\hline \multicolumn{4}{|l|}{ Demographic } \\
\hline Med age (min-max) & $45(26-58)$ & $28(20-69)^{\mathrm{a}}$ & $46(27-78)^{a}$ \\
\hline $\begin{array}{l}\text { Male participant } \\
\text { frequency (\%) }\end{array}$ & $70.6^{b, c}$ & $27.3^{\mathrm{b}}$ & $26.3^{c}$ \\
\hline Smoker frequency (\%) & 76.5 & 72.7 & 63.2 \\
\hline \multicolumn{4}{|l|}{ Clinical Diagnosis*,$n$} \\
\hline Active PTB & 16 & 0 & 0 \\
\hline $\begin{array}{l}\text { PTP w/ extrapulmonary } \\
\text { TB }\end{array}$ & 1 & 0 & 0 \\
\hline LRTI & 0 & 0 & 7 \\
\hline Malignancy** & 0 & 0 & 6 \\
\hline COPD & 2 & 0 & 6 \\
\hline Interstitial lung disease & 0 & 0 & 1 \\
\hline \multicolumn{4}{|l|}{ Comorbidities } \\
\hline DM & 2 & 0 & 2 \\
\hline Rheumatoid arthritis & 0 & 0 & 1 \\
\hline HIV infection & 1 & 0 & 6 \\
\hline
\end{tabular}

* Participants from each group (TB, Healthy and OLD) may present with multiple clinical diagnoses and syndromes

** Includes all forms of malignancies

${ }^{a}$ indicates significant differences between age and cohort. Significance determined at $P<0.05$ using and one way ANOVA with Fisher's LSD as post hoc for all pairwise comparisons

${ }^{b}$ and ${ }^{C}$ indicate significant differences between gender and cohort. Significance determined at $P<0.05$ using Fisher's exact test

$T B$ tuberculosis, OLD other lung disease, med median, min minimum, max maximum, \% percent, PTP pulmonary tuberculosis, $w /$ with, LRT/ lower respiratory tract infection, DM diabetes mellitus, HIV human immunodeficiency virus, $C O P D$ chronic obstructive pulmonary disease
Table 2 Average intravenous sedation doses, bronchoscopy duration and incidental findings at the time of bronchoscopy

\begin{tabular}{|c|c|c|c|c|}
\hline & $\begin{array}{l}\text { TB } \\
(n=26)\end{array}$ & $\begin{array}{l}\text { Healthy } \\
(n=12)\end{array}$ & $\begin{array}{l}\text { Contacts } \\
(n=20)\end{array}$ & $\begin{array}{l}\text { All Cohorts } \\
\text { (range) }\end{array}$ \\
\hline \multicolumn{5}{|c|}{ Average intravenous sedation doses } \\
\hline Midazolam (mg) & 6.7 & 0.0 & 5.1 & $5.9(4.0-10.0)$ \\
\hline Fentanyl (mcg) & 33.6 & 27.3 & 35.7 & $32.2(12.5-75.0)$ \\
\hline Propofol (mg) & 101.8 & 103.3 & 83.4 & $96.1(30.0-250.0)$ \\
\hline \multicolumn{5}{|c|}{ Average bronchoscopy duration } \\
\hline Time (minutes) & 13.0 & 11.3 & 12.6 & $12.0(7.0-30.0)$ \\
\hline \multicolumn{5}{|l|}{ Incidental findings $(n)$} \\
\hline Vocal cord nodule & 1 & 0 & 0 & $1(0-1)$ \\
\hline Vocal cord polyp & 0 & 0 & 1 & $1(0-1)$ \\
\hline Bronchial lesions & 2 & 0 & 0 & $2(0-2)$ \\
\hline
\end{tabular}

$T B$ tuberculosis, Healthy healthy community controls, Contacts household contacts, $m g$ milligrams, mcg micrograms

isolation or a combination of fentanyl and propofol. One case received a combination of fentanyl and midazolam and one received only propofol. The total average dose used for each drug within all reported groups was 5.9 $\mathrm{mg}, 32.2 \mathrm{mcg}$ and $96.1 \mathrm{mg}$, for midazolam, fentanyl and propofol, respectively. The average time duration of the bronchoscopy procedure was $12 \mathrm{~min}$ with no substantial difference noted between TB cases and controls $(P=0.76$, Mann-Whitney U test, Table 2).

Of the 58 participants that underwent bronchoscopy, a total of four participants had incidental findings at the time of bronchoscopy (Table 2). Two participants had vocal cord abnormalities, one had a polyp and one a nodule and were referred for further assessment. In two cases, suspicious bronchial lesions were observed. In one case, a tissue sample was obtained, while the other was referred for further workup (Table 2).

\section{Pre- and post-bronchoscopy adverse events}

Table 3 describes adverse events, complications and symptoms experienced during and post bronchoscopy for $\mathrm{TB}$, healthy community controls, $\mathrm{TB}$ contacts, and a combination of all cohorts. None of the participants presented with serious adverse events. Two participants experienced complications during the bronchoscopy procedure. One developed epistaxis, which resolved prior to hospital discharge, and one presented with elevated blood pressure readings prior to, throughout, and post bronchoscopy procedure in a participant not known with hypertension (Table 3 ). In one case within the healthy cohort the bronchoscopy procedure was aborted and no BALF was retrieved due to distress and discomfort experienced by the participant, despite administration of sedative drugs (Fig. 1). At the $72 \mathrm{~h}$ post bronchoscopy telephonic follow up, 56 of the 58 participants were contactable. A sore throat was the most 
Table 3 Complications during bronchoscopy and post bronchoscopy symptoms at $72 \mathrm{~h}$ follow-up

\begin{tabular}{|c|c|c|c|}
\hline Symptom & $\begin{array}{l}\text { During } \\
\text { bronchoscopy } \\
(n=58)\end{array}$ & $\begin{array}{l}72 \text { h Post } \\
\text { bronchoscopy } \\
\left({ }^{a} n=56\right)\end{array}$ & $\begin{array}{l}\text { Participant } \\
\text { Group/s }\end{array}$ \\
\hline Sore throat & 0 & $6(11 \%)$ & $\begin{array}{l}2 \text { TB, } 2 \text { Healthy, } \\
2 \text { Contacts }\end{array}$ \\
\hline Vomiting & 0 & $1(2 \%)$ & 1 Healthy \\
\hline Haemoptysis & 0 & 0 & - \\
\hline Epistaxis & $1(2 \%)$ & 0 & 1 Healthy \\
\hline $\mathrm{SOB}$ & 0 & 0 & - \\
\hline Cough & 0 & 0 & - \\
\hline Dizziness & 0 & $1(2 \%)$ & $1 \mathrm{~TB}$ \\
\hline Elevated BP & $1(2 \%)$ & 0 & $1 \mathrm{~TB}$ \\
\hline${ }^{\mathrm{b}}$ Discomfort & $1(2 \%)$ & 0 & 1 Healthy \\
\hline Total & $3(5 \%)$ & $8(14 \%)$ & $\begin{array}{l}4 \mathrm{~TB}, 5 \text { Healthy, } \\
2 \text { Contacts }\end{array}$ \\
\hline
\end{tabular}

a at 72 -h follow-up, 56 of 58 participants were contactable

${ }^{b}$ bronchoscopy aborted due to participant discomfort, no BALF collected, participant followed up

$T B$ tuberculosis, Healthy healthy community control/s, Contacts household contacts, hrs hours, \% percentage, SOB shortness of breath, BP blood pressure

common bronchoscopy-related symptom reported. Six participants (11\%) experienced a sore throat, one of which also presented with a single episode of vomiting; and one case reported dizziness; however, symptoms were resolved by the day 14 telephonic follow up. At the day 14 telephonic follow up, only one participant presented with a new symptom namely, a sore throat. None of the participants required medical attention or hospital admission for their symptoms (Table 3).

\section{Tolerance and experience of the procedure}

Participants from the TB $(n=10)$ and healthy community control $(n=12)$ cohorts completed a questionnaire relating to their bronchoscopy experience, as described in Fig. 2, of which all 22 agreed to partake in the questionnaire. The majority of participants, $(n=18,82 \%)$ agreed that the bronchoscopy procedure was tolerable
(Fig. 2a). Twenty (91\%) participants reported the bronchoscopy to be not as bad as expected (Fig. 2b) and 18 (82\%) participants reported willingness to undergo another research bronchoscopy in future (Fig. 2c). Reasons provided for not wanting to undergo a second research bronchoscopy included discomfort and anxiety related to the procedure and overall experience.

\section{BALC characteristics: differential cell counts}

BALF samples were retrieved from $17 \mathrm{~TB}$ cases, 11 healthy community controls and 19 OLD participants and used for cell isolation. BALC derived therefrom were evaluated by differential cell count analysis using light microscopy and differential staining techniques (Table 4 and Additional file 1: Figure S2; subject characteristics in Table 1).

Statistical analysis was performed in order to evaluate whether there was a relationship between the colour of the BALC pellet and smoking status of study participants. Black BALC pellets were predominant in the smoking population $(P=0.01)$ (Fig. 3 ; also see Fig. 5 ).

Significant changes in differential BALC counts were also considered in the context of potential co-variates such as pellet colour, smoking, endoscopist, gender or age (Table 1, Additional file 2: Figure S1). There was a significant difference in the number of bronchoscopies performed per endoscopist $(P=0.02$, Additional file 2 : Figure S1a), meaning that each endoscopist did not perform the procedure on the same number of patients in each cohort. However, smoking status $(P=0.21$, Additional file 2: Figure S1b) and pellet colour $(P=0.69$, Additional file 2: Figure S1c) show no statistically significant differences between cohorts. We report males being more likely to have $\mathrm{TB}$, relative to females $(P=0.02$, Additional file 2: Figure S1d, Table 1). Additionally, results show age of healthy community controls to be significantly lower than the OLD cohort $(P=0.01$, Additional file 2: Figure S1e, Table 1).

It was also of interest to determine variations in cellular data readouts between cohorts including, retrieved
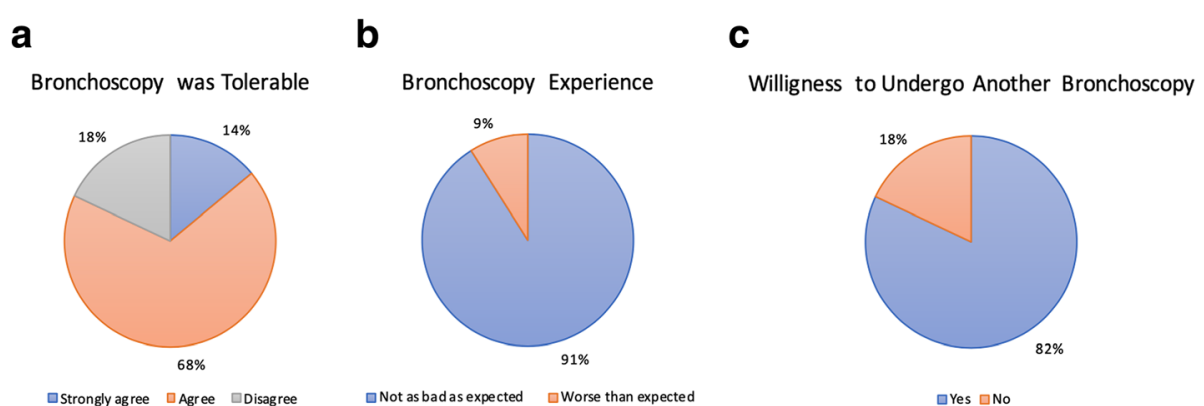

Fig. 2 a Tolerability, b) experience, and c) willingness to undergo another bronchoscopy, reported subjectively by participants. A total of $n=22$ participants completed a telephonic questionnaire, including $n=10$ TB and $n=12$ healthy community controls 
Table 4 Evaluation of BALF and BALC form study participants

\begin{tabular}{|c|c|c|c|}
\hline & $\mathrm{TB}(n=17)$ & Healthy $(n=11)$ & $\operatorname{OLD}(n=19)$ \\
\hline \multicolumn{4}{|l|}{ Saline and BALF } \\
\hline Med saline volume (ml) in (min-max) & $190(60-300)$ & $170(120-240)$ & $180(120-300)$ \\
\hline Med BALF volume (ml) out (min-max) & $65(40-125)$ & $90(45-135)$ & $90(37-155)$ \\
\hline Fluid volume ratio out/in (min-max) & $0.31(0.17-0.67)$ & $0.54(0.20-0.67)$ & $0.49(0.12-0.86)$ \\
\hline \multicolumn{4}{|l|}{ Cellular (based on differential cell counts) } \\
\hline Cell pellet colour (Black/Clear) & $12 / 5$ & $7 / 4$ & $8 / 11$ \\
\hline Total med BALC count $\left(\times 10^{6}\right)$ (min-max) & $38.00(26.40-54.00)^{\mathrm{a}}$ & $21.15(11.25-81.60)$ & $16.20(6.50-43.40)^{a}$ \\
\hline Med BALC concentration $\left(\times 10^{6} / \mathrm{ml}\right)$ (min-max) & $0.68(0.10-1.39)$ & $0.44(0.14-1.31)$ & $0.49(0.12-0.86)$ \\
\hline Med \% Monocytes/Macrophages (min-max) & $81.00(0.00-88.00)^{b, c}$ & $89.00(70.00-98.00)^{b}$ & $80.50(5.00-98.00)^{c}$ \\
\hline Med \% Neutrophils (min-max) & $10.00(2.00-100.00)^{\mathrm{d}, \mathrm{e}}$ & $0.00(0.00-4.00)^{d}$ & $9.00(0.00-21.00)^{e}$ \\
\hline Med \% Lymphocytes (min-max) & $9.00(0.00-13.00)^{f}$ & $11.00(2.00-30.00)^{f}$ & $7.00(0.00-30.00)$ \\
\hline
\end{tabular}

${ }^{\text {a-f }}$ Illustrates statistical differences of data readouts between cohorts. Significance determined at $P<0.05$ using ANCOVA with Fisher's LSD as post hoc for all pairwise comparisons. Statistical analysis factors in co-variant data of gender and age differences between cohorts

TB tuberculosis, OLD other lung disease, med median, min minimum, max maximum, $\mathrm{m} / \mathrm{millilitre,} \%$ percentage

BALF/instilled saline ratios, cell yields, cell concentrations, macrophage/monocyte frequency, neutrophil frequency and lymphocyte frequency for TB, healthy community control and OLD cohorts (Table 4, Additional file 1: Figure S2). Taking significant co-variant data into account (those being age and gender), we find that BALC yield and cell concentration (Table 4, Additional file 1: Figure S2b-c) was greater in TB participants relative to the OLD cohort $\left(P=4.19 \times 10^{-3}\right.$ and $P=0.05$, respectfully). Macrophage/ monocyte frequencies in the TB cohort are significantly lower than healthy community controls $(P=0.04)$ and OLD participants $(P=0.01)$, with a concomitant increase in neutrophil frequencies in the TB cohort relative to the healthy community controls $\left(P=2.89 \times 10^{-3}\right)$ and OLD patients $\left(P=1.57 \times 10^{-3}\right)$ (Table 4, Additional file 1: Figure S2d-e). We also show lymphocyte frequencies to be significantly lower in TB participants relative to healthy community controls $(P=0.03$, Additional file 1: Figure S2f).

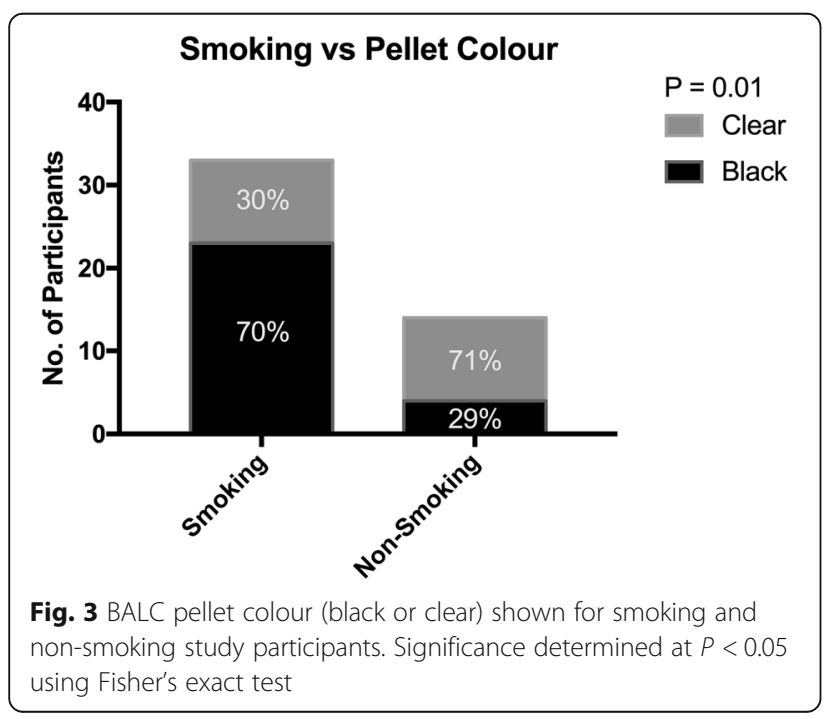

Irrespective of cohort, correlations were investigated between volume ratios and cell yield, cell concentration and age (Additional file 3: Figure S3a-c), as well as correlations between age and cell yield, cell concentration, monocyte/macrophage frequencies, neutrophil frequencies and lymphocyte frequencies (Fig. 4 and Additional file 3: Figure S3d-f). Figure 4 shows significant negative correlative relationship between macrophage/monocyte frequencies and age $(r=-0.40 ; P=0.01)$ and a positive correlative relationship between neutrophil frequencies and age $(r=-0.49 ; P=0.00)$, irrespective of cohort.

We determined that no statistically significant relationships exist between cellular readouts (volume ratio, cell yield, cell concentration, macrophage/monocyte frequency, neutrophil frequency and lymphocyte frequency) and factors such as pellet colour (Additional file 4: Figure S4), gender (Additional file 5: Figure S5) and smoking status (Additional file 6: Figure S6).

\section{BALC characteristics: flow cytometric analyses}

The flow cytometer was calibrated to account for the background fluorescence of BALC. BALC from smokers, demonstrating black BALC pellets (Fig. 5), displayed significant autofluorescence (Fig. 6b), when compared to the non-smoker group with clear BALC (Fig. 6a). BALC autofluorescence interfered with the detected antibody fluorescence signal in unquenched and quenched samples (Fig. 6). Fluorescence spill-over was observed in all investigated channels, complicating accurate assessment of cell classifications and frequencies of innate cell types (data not shown).

\section{Discussion}

According to the data presented here, bronchoscopies with BAL can be considered a safe procedure (outweighing the benefit-to-risk ratio) with no major complications 

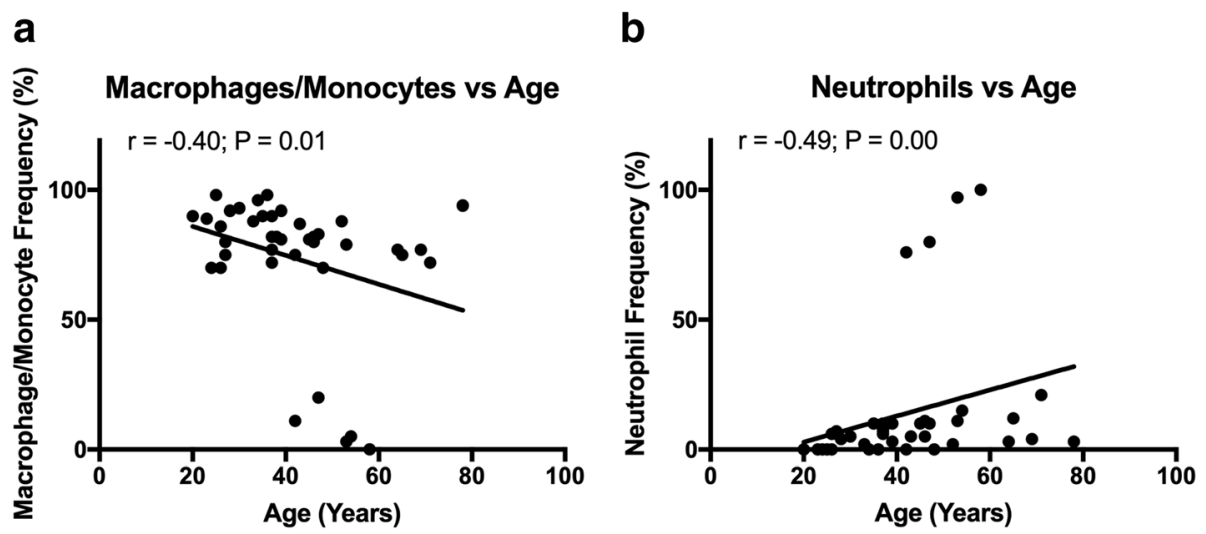

Fig. 4 Correlative relationships between a) macrophage frequency and age, b) neutrophil frequency and age. Significance determined at $P<0.05$ using Pearson's correlation or Spearman's rank-order correlation tests, as appropriate following normality testing

or adverse events evident in either healthy or diseased participants included in this study. Of those partaking in the post-bronchoscopy survey, $82 \%$ of participants described the procedure as tolerable, with an estimated repeatability rate of $82 \%$. Only $14 \%$ of participants presented with minor post bronchoscopy symptoms, mainly reports of a sore throat. These data are comparable to known literature reporting similar repeatability rates, although Lipman et al. demonstrated that a higher proportion of participants $(80 \%)$ experienced post bronchoscopy symptoms such as a sore throat [8]. This is likely due to a later evaluation timepoint in our study ( $72 \mathrm{~h}$ vs $24 \mathrm{~h}$ ), when some symptoms may have already subsided. However, this observation may also be influenced by cultural backgrounds and health-seeking behavior. Furthermore, the average bronchoscopy procedural time reported by our findings, from insertion to removal of the bronchoscope, compared well with a study performed by Grendelmeier et al. [15].

In terms of cellular data derived from BALF, we describe a strong relationship between pellet colour and smoking habits, with smoking being associated with yields of black
BALC that strongly interfere with fluorescence-based flow cytometry analysis techniques. This phenomenon has previously been described $[16,17]$. In light of these findings, we propose an important need for fluorescenceindependent techniques for quantitative phenotypic analysis of such samples.

Participants for this study were recruited from lower income communities of the Western Cape province, with the highest smoking prevalence and TB burden in South Africa [18]. In line with previously reported statistics and our findings reported here, we suggest that BAL-derived cellular analysis from smoking participants of the Western Cape population is not suitable for fluorescence-based techniques and propose further investigation into the impact of smoking on host control of mycobacterial infection at the disease site in the current population. We report a higher rate of males (70.6\%) and smokers (76\%) comprising the TB cohort, relative to healthy community controls and OLD cohorts, which are indeed hallmark characteristics that have been previously reported for TB patients in middle-

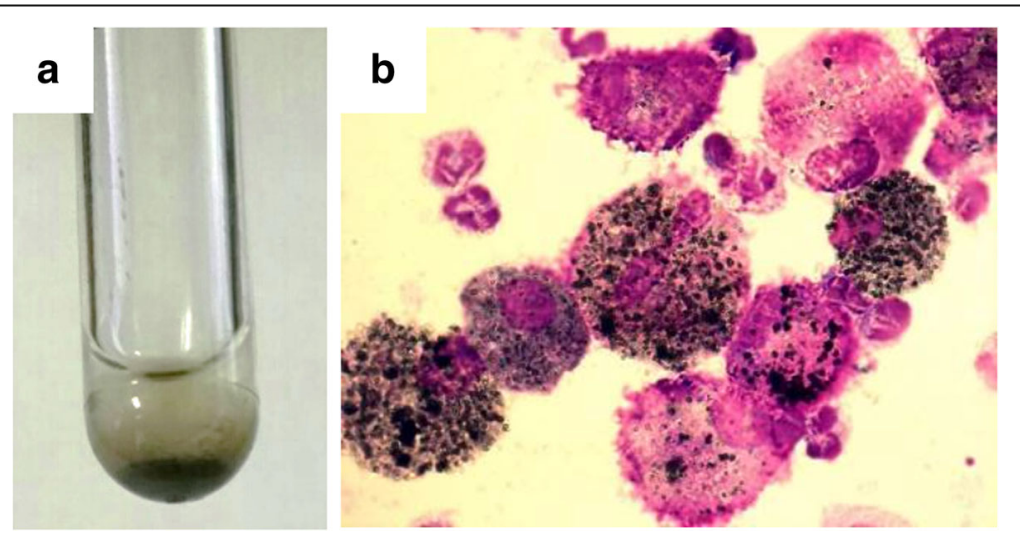

Fig. 5 Exposure to carbonaceous particles from biofuel burning. a Carbon loading was visible as blackened BALC pellets. b Particles were mainly contained in alveolar macrophages of blackened BALC 

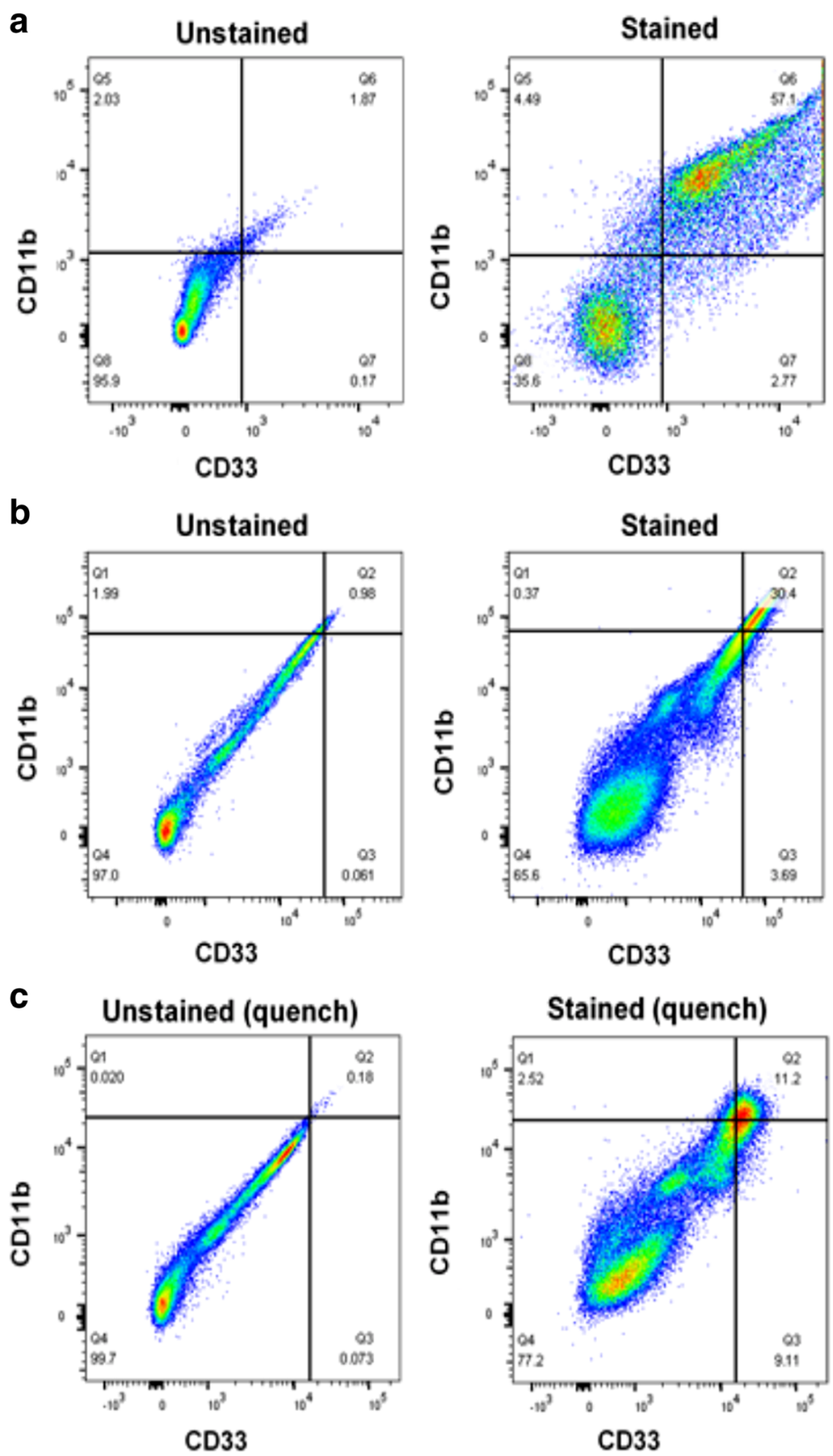

Fig. 6 Identification of myeloid cells from BALC samples of TB patients. FSC and SSC characteristics were selected to define all BALC. All subsequent analyses were performed on cells selected in the BALC gate. a Gating strategy used to define CD11b + CD33+ innate cells from BALC with low autofluorescence signal. b Gating strategy selected to define CD11b + CD33+ innate cells from BALC presenting with extensive autofluorescence. c Gating strategy identifying CD11b + CD33+ innate cells from the same BALC indicated in (b), post quenching treatment

to low-income communities in South Arica $[6,19,20]$. Our data also show a significant difference in age between cohorts. As such, we report sex and age as covariables that are controlled and adjusted for when reporting differences in cellular data between cohorts. We report no significant differences of smoking status and pellet colour between cohorts. This study was not powered to fully evaluate the propensity for variation introduced by different endoscopists performing the bronchoscopy.

Although no significant differences were measured for fluid volume ratio and cell concentration between cohorts, total cell yield was increased in the TB cohort relative to OLD participants. In terms of cellular 
composition of the BALF, we report macrophages/ monocytes to be the predominant BALC population, with frequencies above $80 \%$ for all cohorts, which is in line with extensive publications reporting macrophages as the major cell lineage within the BALF [21-24]. Furthermore, we report TB disease to be characterized by increased neutrophil frequencies and decreased proportions of lymphocytes and macrophages/monocytes in the BALC compartment. Neutrophils have been described to be increased and predominant in Mtb-infected regions of the lung in TB patients, with many studies reporting neutrophilic infiltration and exorbitant inflammation in TB disease that is considered to be an overall reflection of TB disease severity [25-30]. The decrease in macrophage/monocyte frequencies in the TB cohort may reflect increased numbers and proportions of neutrophilic populations that predominate in $\mathrm{TB}$ patients and thus translate into decreased macrophage frequency expressed as a percentage. Alternatively, we could argue in favour of previously published data that supports the observed decreased frequency of alveolar macrophage frequencies in the BALF of TB-infected patients [25, 31, 32]. Another study proposed the ability of Mtb to hijack macrophages and promote translocation from the airway to the lung parenchyma, thus acting as supporting evidence to substantiate the reported pattern of decreased frequencies and cell number of airway macrophages within the BALF of TB-laden individuals, relative to the appropriate controls [33]. Contrary to previous publications that report an influx of lymphocytes to the site of TB disease, we report that lymphocytes are reduced in TB participants and OLD, relative to healthy controls [25, 34, 35]. Notably, multiple independent studies have reported a significant tendency of BALC analysis by optical microscopy to underestimate the percentage of lymphocytes and overestimate the frequency of macrophages in the BAL, with common misinterpretations of stained BAL specimens due to the propensity of activated cells to resemble other immune cell lineages [36-40]. In light of these findings, we conclude discrepant BALC frequencies to previously reported BALC populations using microscopy-based differential cell counting, suggesting the value of more accurate phenotyping approaches such as definitive antibody-based cell labelling techniques.

We also report that macrophages have a significant negative relationship with age of the participant, while neutrophils have a positive relationship with participant age. This observation is reported irrespective of participant cohort, however, since macrophage/monocyte and neutrophil frequencies show significant differences between cohorts, the significance of the correlative data may be influenced by different participant groups. We propose that these findings require validation using more accurate phenotyping and quantification techniques. Our results further suggest that black BALC pellets (and the related tendency to smoking) are characterized by an increase in macrophage/monocyte populations in the airways relative to non-smokers, which is in line with several other studies [41-44]. In line with our findings, several studies have reported no gender-related differences in BALC composition [45-47].

Limitations to this study include the relatively small participant group enrolled in this study. We recommend that the experience questionnaire and cellular data comparisons between population groups be studied in a bigger cohort in order to better capture data representing the population at large. We also acknowledge that this study was limited in its power to determine the effect of endoscopist variability on cellular data outcomes, which may certainly be a cofounding factor, depending on experience, level of expertise and personal preferences in bronchoscopy proceedings that may lead to variability in the dataset. Finally, we acknowledge the propensity for human error and subjective interpretation of microscopy-based differential counting and highlight the need for further investigations into BALC characteristics using more objective, accurate means of cellular phenotyping, using fluorescence-independent techniques.

\section{Conclusions}

We report significantly increased BALC yields, decreased macrophage/monocyte frequencies, increased neutrophil frequencies and decreased lymphocyte frequencies in $\mathrm{TB}$ patients, relative to healthy and OLD control cohorts. Furthermore, macrophage/monocyte frequencies are significantly negatively correlated with age, while neutrophils are significantly positively correlated with age, irrespective of cohort. We report that smoking is characterized by black BALC yields and indeed interferes with fluorescence-based analyses. The data presented here could be beneficial to researchers designing studies involving samples derived from bronchoscopy procedures in the Western Cape population. Researchers may use these data as a basis for planning, designing and anticipating the outcomes of research bronchoscopies from the point of participant recruitment through to BALF processing and cellular readouts of retrieved samples, which is certainly a valuable research tool in highly TB-burdened communities where extensive research is warranted.

\section{Additional files}

Additional file 1: Figure S2. Comparison of BALC data readouts between cohorts. Data readouts: a) volume ratio, b) cell yield, c) cell concentration, d) macrophage frequency, e) neutrophil frequency and f) lymphocyte frequency. Significance determined at $P<0.05$ and shown in 
red, using ANCOVA with Fisher's LSD as post hoc for all pairwise comparisons. (PNG $263 \mathrm{~kb}$ )

Additional file 2: Figure S1. The relationship between TB, healthy community control and OLD cohorts and potential co-variables: a) endoscopist, b) smoking status, and c) pellet colour, d) gender and e) age; that may affect cellular BAL data comparisons between cohorts. Significance determined at $P<0.05$, using Fisher's exact test (a-d) and one-way ANOVA with Fisher's LSD as post hoc for all pairwise comparisons (e). (PNG $334 \mathrm{~kb}$ )

Additional file 3: Figure $\mathbf{S 3}$. Correlative relationships between a) volume ratio and cell yield, b) volume ratio and cell concentration, c) volume ratio and age, d) cell yield and age, e) cell concentration and age, $f$ ) lymphocyte frequency and age. Significance determined at $P<0.05$, using Pearson's correlation or Spearman's rank-order correlation tests, as appropriate following normality testing. (PNG $319 \mathrm{~kb}$ )

Additional file 4: Figure S4. The relationship between BALC pellet colour and a) volume ratio, b) cell yield, c) cell concentration, d) monocyte frequency, e) neutrophil frequency, and f) lymphocyte frequency. Significance determined at $P<0.05$, using one-way ANOVA tests (parametric or non-parametric as appropriate after normality test and Levene's test for homogeneity of variance) with Fisher's least significant difference (LSD) as post host for all pairwise comparisons. (PNG $243 \mathrm{~kb}$ )

Additional file 5: Figure S5. The relationship between participant gender and a) volume ratio, b) cell yield, c) cell concentration, d) monocyte frequency, e) neutrophil frequency, and f) lymphocyte frequency. Significance determined at $P<0.05$, using one-way ANOVA tests (parametric or non-parametric as appropriate after normality test and Levene's test for homogeneity of variance) with Fisher's least significant difference (LSD) as post host for all pairwise comparisons. (PNG $240 \mathrm{~kb}$ )

Additional file 6: Figure S6. The relationship between participant smoking status and a) volume ratio, b) cell yield, c) cell concentration, d) monocyte frequency, e) neutrophil frequency, and f) lymphocyte frequency. Significance determined at $P<0.05$, using one-way ANOVA tests (parametric or non-parametric as appropriate after normality test and Levene's test for homogeneity of variance) with Fisher's least significant difference (LSD) as post host for all pairwise comparisons. (PNG 274 kb)

\section{Abbreviations}

ANCOVA: Analysis of co-variance; ANOVA: Analysis of variance; BAL: Bronchoalveolar lavage; BALC: Bronchoalveolar lavage cells; BALF: Bronchoalveolar lavage fluid; CRF: Clinical research form; CXR: Chest Xray; DM: Diabetes mellitus; ECG: Electrocardiogram; FCS: Fetal calf serum; HIV: Human immunodeficiency virus; LSD: Least significant difference; Mtb: Mycobacterium tuberculosis; OLD: Other lung diseases; PBS: Phosphate buffered saline; PTB: Pulmonary tuberculosis; STARD: Standards for reporting of diagnostic accuracy; TB: Tuberculosis; TBH: Tygerberg hospital

\section{Acknowledgements}

The authors acknowledge the study participants and the Stellenbosch University-Immunology Research Group (SU-IRG) Consortium: Coenraad F.N. Koegelenberg; Léanie Kleynhans; Katharina Ronacher; Jane A. Shaw, Donald Simon, Shirley McAnda, Katherina C. Swartz.

\section{Authors' contributions}

The manuscript was written and developed by all authors. All authors read and approved the final manuscript.

\section{Funding}

The authors declare and acknowledge the financial support from the European \& Developing Countries Clinical Trials Partnership (EDCTP; CDF1546) and International Collaborations in Infectious Disease Research (ICIDR): Biology and Biosignatures of anti-TB Treatment Response (5U01/A115619/03) and the National Institute of Health (R01Al116039). The authors acknowledge support from the South-African Department of Science and Technology (SA-DST) and South-African National Research Foundation (SA-NRF) through the South African Research Chair Initiative (SARChi) in TB Biomarkers (\#86535).

\section{Availability of data and materials}

The datasets used and/or analysed during the current study are available from the corresponding author on reasonable request.

\section{Ethics approval and consent to participate}

Written informed consent was obtained from participants and study design was approved by the Stellenbosch University Ethics Review Committee (IRB number N16/05/070 and N13/05/064).

\section{Consent for publication}

Not applicable.

\section{Competing interests}

The authors declare that the literature review and perspectives was performed in the absence of any commercial or financial relationships that could be construed as potential conflict of interest.

Received: 3 April 2019 Accepted: 24 May 2019

Published online: 01 June 2019

\section{References}

1. Meyer KC. Bronchoalveolar lavage as a diagnostic tool. Semin Respir Crit Care Med. 2007;28:546-60.

2. Balbi B, Pignatti $P$, Corradi M, Baiardi $P$, Bianchi L, Brunetti G, et al. Bronchoalveolar lavage, sputum and exhaled clinically relevant inflammatory markers: values in healthy adults. Eur Respir J. 2007:30(4):769-81.

3. Reynolds HY, Fulmer JD, Kazmierowski JA, Roberts WC, Frank MM, Crystal RG. Analysis of cellular and protein content of broncho-alveolar lavage fluid from patients with idiopathic pulmonary fibrosis and chronic hypersensitivity pneumonitis. J Clin Invest. 1977;59(1):165-75.

4. Costabel U, Guzman J. Bronchoalveolar lavage in interstitial lung disease. Curr Opin Pulm Med. 2001 Sep;7(5):255-61.

5. Heron M, Grutters JC, ten Dam-Molenkamp KM, Hijdra D, van HeugtenRoeling A, Claessen AME, et al. Bronchoalveolar lavage cell pattern from healthy human lung. Clin Exp Immunol. 2012;167(3):523-31.

6. World Health Organization. Global tuberculosis report 2018. 2018.

7. Poi PJ, Chuah SY, Srinivas P, Liam CK. Common fears of patients undergoing bronchoscopy. Eur Respir J. 1998;11(5):1147-9.

8. Lipman MC, Stobbs D, Madge S, Miller R, Johnson MA. Research bronchoscopies do not adversely affect HIV-infected individuals' future health-care decisions. Chest. 1998;114(1):284-90.

9. Martinsen EMH, Leiten EO, Bakke PS, Eagan TML, Grønseth R. Participation in research bronchoscopy: a literature review. Eur Clin Respir J. 2016;3(1):29511.

10. Babb J, Bowie P, Brewin A, Fraise A, Garrard C, Harvey J, et al. British Thoracic Society guidelines on diagnostic flexible bronchoscopy. Thorax. 2001 Mar;56(Suppl 1):i1-21.

11. Meyer KC, Raghu G, Baughman RP, Brown KK, Costabel U, Du Bois RM, et al. An official American Thoracic Society clinical practice guideline: the clinical utility of bronchoalveolar lavage cellular analysis in interstitial lung disease. Am J Respir Crit Care Med. 2012;185(9):1004-14.

12. Strober W. Trypan blue exclusion test of cell viability. Curr Protoc Immunol. 1997;21(1):A. 3B. 1-2

13. Veenstra $H$, Dowdle EB. Multi-well cell monolayers for immunocytochemistry. An alternative to cytocentrifuge preparations. J Immunol Methods. 1992:146(2):257-8.

14. Hallden G, Sköld CM, Eklund A, Forslid J, Hed J. Quenching of intracellular autofluorescence in alveolar macrophages permits analysis of fluorochrome labelled surface antigens by flow cytofluoremetry. J Immunol Methods. 1991:142(2):207-14.

15. Grendelmeier P, Tamm M, Pflimlin E, Stolz D. Propofol sedation for flexible bronchoscopy: a randomised, noninferiority trial. Eur Respir J. 2014;43(2):591-601

16. Reddy P, Meyer-Weitz A, Yach D. Smoking status, knowledge of health effects and attitudes towards tobacco control in South Africa. S Afr Med J. 1996;86(11):1389-93.

17. den BS, van LSWP, Borgdorff MW, Verver S, Bateman ED, Lombard CJ, et al. Association between smoking and tuberculosis infection: a population survey in a high tuberculosis incidence area. Thorax. 2005:60(7):555-7.

18. Reddy P, Zuma K, Shisana O, Jonas K, Sewpaul R. Prevalence of tobacco use among adults in South Africa: results from the first south African National Health and nutrition examination survey. S Afr Med J. 2015;105(8):648-55. 
19. Lin $\mathrm{H}-\mathrm{H}$, Ezzati M, Murray M. Tobacco smoke, indoor air pollution and tuberculosis: a systematic review and meta-analysis. PLoS Med. 2007;4(1):e20.

20. Bates MN, Khalakdina A, Pai M, Chang L, Lessa F, Smith KR. Risk of tuberculosis from exposure to tobacco smoke: a systematic review and meta-analysis. Arch Intern Med. 2007;167(4):335-42.

21. Green GM, Kass EH. The role of the alveolar macrophage in the clearance of bacteria from the lung. J Exp Med. 1964;119(1):167-76.

22. Hirayama $D$, lida $T$, Nakase $H$. The phagocytic function of macrophageenforcing innate immunity and tissue homeostasis. Int J Mol Sci. 2017;19(1):92.

23. Rubins JB. Alveolar macrophages: wielding the double-edged sword of inflammation. Am J Respir Crit Care Med. 2003;167(2):103-4.

24. Sibille Y, Reynolds HY. Macrophages and Polymorphonuclear neutrophils in lung defense and Injury1-2. Am Rev Respir Dis. 1990;141:471-501.

25. Ozaki T, Nakahira S, Tani K, Ogushi F, Yasuoka S, Ogura T. Differential cell analysis in bronchoalveolar lavage fluid from pulmonary lesions of patients with tuberculosis. Chest. 1992;102(1):54-9.

26. Eum S-Y, Kong J-H, Hong M-S, Lee $Y-J$, Kim J-H, Hwang S-H, et al. Neutrophils are the predominant infected phagocytic cells in the airways of patients with active pulmonary TB. Chest. 2010;137(1):122-8.

27. Greco S, Marruchella A, Massari M, Saltini C. Predictive value of BAL cellular analysis in differentiating pulmonary tuberculosis and sarcoidosis. Eur Respir J. 2005;26(2):360-2

28. Barry S, Breen R, Lipman M, Johnson M, Janossy G. Impaired antigen-specific CD4+ T lymphocyte responses in cavitary tuberculosis. Tuberculosis. 2009; 89(1):48-53.

29. Ong CW, Elkington PT, Brilha S, Ugarte-Gil C, Tome-Esteban MT, Tezera LB, et al. Neutrophil-derived MMP-8 drives AMPK-dependent matrix destruction in human pulmonary tuberculosis. PLoS Pathog. 2015;11(5):e1004917.

30. Choi H, Chon HR, Kim K, Kim S, Oh K-J, Jeong SH, et al. Clinical and laboratory differences between lymphocyte-and neutrophil-predominant pleural tuberculosis. PLoS One. 2016;11(10):e0165428.

31. Sharma SK, Pande JN, Verma K. Bronchoalveolar lavage (BAL) in miliary tuberculosis. Tubercle. 1988;69(3):175-8.

32. Mazzarella G, Bianco A, Perna F, D'AURIA D, Grella E, Moscariello E, et al. T lymphocyte phenotypic profile in lung segments affected by cavitary and non-cavitary tuberculosis. Clin Exp Immunol. 2003;132(2):283-8.

33. Cohen SB, Adams K, Urdahl K. Mycobacterium tuberculosis hijacks alveolar macrophages to translocate from the airway to the lung parenchyma. Cell Host Microbe. 2018;24(3):439-46 e4.

34. Hoheisel GB, Tabak L, Teschler H, Erkan F, Kroegel C, Costabel U. Bronchoalveolar lavage cytology and immunocytology in pulmonary tuberculosis. Am J Respir Crit Care Med. 1994;149(2):460-3.

35. Raju B, Tung CF, Cheng D, Yousefzadeh N, Condos R, Rom WN, et al. In situ activation of helper T cells in the lung. Infect Immun. 2001;69(8):4790-8.

36. Tricas L, Echeverría A, Blanco MA, Menéndez M, Belda J. Flow cytometry counting of bronchoalveolar lavage leukocytes with a new profile of monoclonal antibodies combination. Cytometry B Clin Cytom. 2012;82(2):61-6.

37. Barry SM, Condez A, Johnson MA, Janossy G. Determination of bronchoalveolar lavage leukocyte populations by flow cytometry in patients investigated for respiratory disease. Cytom J Int Soc Anal Cytol. 2002;50(6):291-7.

38. Hodge SJ, Hodge GL, Holmes M, Reynolds PN. Flow cytometric characterization of cell populations in bronchoalveolar lavage and bronchial brushings from patients with chronic obstructive pulmonary disease. Cytom Part B Clin Cytom J Int Soc Anal Cytol. 2004;61(1):27-34.

39. Arias M, Palomo B, Belda J, Tricas L, Paniagua MP, Martinez C, et al. Differential cell count in BAL by flow cytometry using CD15 FITC/ CD16PE/CD45 PERCP/HLA-DR APC monoclonal antibodies. Eur Respir Soc. 2011;38:4770

40. Robinson-Smith TM, Saad A, Baughman RP. Interpretation of the Wright-Giemsa stained bronchoalveolar lavage specimen. Lab Med. 2004;35(9):553-7.

41. Selvaraj P, Venkataprasad N, Vijayan VK, Prabhakar R, Narayanan PR. Alveolar macrophages in patients with pulmonary tuberculosis. Lung India. 1988;6(2):71-4.

42. Burke WM, Roberts CM, Bryant DH, Cairns D, Yeates M, Morgan GW, et al. Smoking-induced changes in epithelial lining fluid volume, cell density and protein. Eur Respir J. 1992;5(7):780-4.
43. Jimenez CR, Rajas O, Ruiz A, Ramos A, Florez S, Ramos L, et al. Bronchoalveolar lavage in smokers: quantification of alveolar macrophages and neutrophils as markers of bronchial obstruction. Vivo Athens Greece. 1998;12(4):427-30.

44. Costabel U, Bross K, Reuter C, Rühle K-H, Matthys H. Alterations in immunoregulatory T-cell subsets in cigarette smokers: a phenotypic analysis of bronchoalveolar and blood lymphocytes. Chest. 1986;90(1):39-44.

45. Ettensohn DB, Jankowski MJ, Duncan PG, Lalor PA. Bronchoalveolar lavage in the normal volunteer subject: I. technical aspects and intersubject variability. Chest. 1988;94(2):275-80.

46. Mund E, Christensson B, Larsson K, Grönneberg R. Sex dependent differences in physiological ageing in the immune system of lower airways in healthy non-smoking volunteers: study of lymphocyte subsets in bronchoalveolar lavage fluid and blood. Thorax. 2001;56(6):450-5.

47. Karimi R, Tornling G, Grunewald J, Eklund A, Sköld CM. Cell recovery in bronchoalveolar lavage fluid in smokers is dependent on cumulative smoking history. PLoS One. 2012;7(3):e34232.

\section{Publisher's Note}

Springer Nature remains neutral with regard to jurisdictional claims in published maps and institutional affiliations.

Ready to submit your research? Choose BMC and benefit from:

- fast, convenient online submission

- thorough peer review by experienced researchers in your field

- rapid publication on acceptance

- support for research data, including large and complex data types

- gold Open Access which fosters wider collaboration and increased citations

- maximum visibility for your research: over $100 \mathrm{M}$ website views per year

At BMC, research is always in progress.

Learn more biomedcentral.com/submissions 